\title{
Importancia de la forma química y de la matriz del sustrato en la toxicidad por cobre en Noticastrum sericeum (Less.) Less. ex Phil.
}

\author{
Importance of the chemical form and type of substrate for copper toxicity in \\ Noticastrum sericeum (Less.) Less. ex Phil.
}

ROSANNA GINOCCHIO ${ }^{1} \&$ JÉSSICA NARVÁEZ ${ }^{2}$

\begin{abstract}
${ }^{1}$ Departamento de Ecología, Pontificia Universidad Católica de Chile, Alameda 340, Santiago, Chile; e-mail: erginocc@genes.bio.puc.cl

${ }^{2}$ Departamento de Química, Facultad de Ciencias, Universidad de Chile, Las Palmeras 3425, Casilla 653, Santiago, Chile
\end{abstract}

\begin{abstract}
RESUMEN
El aumento de la concentración de cobre en los suelos debido a distintas fuentes antrópicas puede producir efectos de toxicidad en las especies vegetales sensibles. Sin embargo, se ha postulado que diversos factores edáficos podrían determinar la biodisponibilidad del cobre y, por lo tanto, la intensidad del efecto fitotóxico, tales como el contenido de materia orgánica y el $\mathrm{pH}$ del suelo, además del grado de solubilidad de la forma química en la cual el cobre es incorporado. Para determinar la importancia de la capacidad tamponante del sustrato de crecimiento y de la forma química del cobre en el grado de fitotoxicidad, se expuso plántulas de una especie sensible a concentraciones crecientes de cobre, incorporado como una sal muy soluble en agua $\left(\mathrm{CuSO}_{4}\right)$ o como una sal muy poco soluble en agua $(\mathrm{CuO})$, a un sustrato sólido altamente tamponante, rico en materia orgánica y de $\mathrm{pH}$ neutro (compost comercial) y a una solución poco tamponante de 2 mmoles $\mathrm{L}^{-1}$ de nitrato de calcio, sin materia orgánica y moderadamente ácida. Se detectaron efectos significativos de toxicidad por cobre en las raíces de las plántulas cultivadas en el sustrato líquido, independientemente de la forma química y de la concentración adicionadas, expresados por una inhibición en el crecimiento de las raíces primarias y en el número de raíces secundarias formadas. Como se esperaba, los efectos de toxicidad fueron menos marcados en el vástago, pero el $\mathrm{CuSO}_{4}$ inhibió significativamente la producción de hojas nuevas en las plantas crecidas en solución, independientemente de la concentración, mientras que el CuO sólo redujo significativamente la producción de hojas al ser agregado a alta concentración. No se detectó efectos de toxicidad en las plantas crecidas en el sustrato sólido, independientemente de la sal de cobre y de la concentración adicionadas, debido a que su alto contenido de materia orgánica y $\mathrm{pH}$ neutro resultaron en una baja disponibilidad de iones cobre para ser absorbidos por las raíces de las plantas. En consecuencia, la respuesta de las plantas a la incorporación de cobre en el sustrato de crecimiento es multifactorial y no puede ser evaluada a partir de los niveles totales de cobre presentes.
\end{abstract}

Palabras clave: biodisponibilidad, fitotoxicidad, contaminación suelos, metales.

\begin{abstract}
The increase of copper concentration in soils due to human activities may result in plant toxicity, particularly in sensitive species. However, it has been postulated that some edaphic factors may determine the bioavailable copper fraction in soils and thus the intensity of fitotoxicity, such as the physicochemistry of the substrate, besides the intrinsic solubility of the chemical form in which copper is incorporated to soil. To determine the importance of the metal buffering capacity of the plant-growth substrate and the copper form on the intensity of copper toxicity, seedlings of a sensitive plant species were exposed to increasing copper concentrations, added as a high water soluble form $\left(\mathrm{CuSO}_{4}\right)$ or as a low water soluble form $(\mathrm{CuO})$ to substrates with high (commercial compost, high content of organic matter and neutral $\mathrm{pH}$ ) and low (solution of $2 \mathrm{mmol} \mathrm{L}^{-1}$ calcium nitrate with no organic matter and slightly acidic $\mathrm{pH}$ ) copper buffering capacity. Significant effects of copper toxicity were found in roots of seedlings grown in solutions spiked with either $\mathrm{CuSO}_{4}$ or $\mathrm{CuO}$, at both experimental concentrations, as growth of primary roots was inhibited and the number of secondary roots was reduced. Shoots showed less effects of toxicity as expected, but a significant reduction in the number of leaves was detected on seedlings grown in solutions spiked with $\mathrm{CuSO}_{4}$, at both concentrations, and with high concentration of $\mathrm{CuO}$. No toxicity effects were detected on seedlings grown in commercial compost spiked either with copper sulphate or copper oxide, at any experimental concentration, because the high buffering capacity of the organic matter and the neutral $\mathrm{pH}$ of the substrate resulted in low availability of copper ions for plant uptake. Therefore, plant response to elevated copper concentrations is multifactorial and cannot be only assessed from the total copper levels added to soils.
\end{abstract}

Key words: bioavailability, fitotoxicity, soil pollution, metals. 


\section{INTRODUCCIÓN}

El suelo es una delgada capa ubicada en la interfase entre la litosfera y la atmósfera y constituye un componente básico de los ecosistemas terrestres al suministrar los nutrientes minerales y el agua esenciales para la vida (Allen et al. 1995). Sin embargo, la contaminación del suelo con metales pesados por diversas actividades antrópicas, tales como la minería metálica, la fundición y refinación de minerales y el uso intensivo de fungicidas cúpricos en la agricultura, entre otros, ha resultado en importantes efectos negativos sobre los ecosistemas terrestres impactados (e.g., Alfaro 1988, Dudka et al. 1995, Kim \& Kim 1999, Ginocchio 2000). Estos efectos negativos se deben a la alta persistencia de los metales en el suelo, en comparación con los otros compartimentos de la biosfera, y a la toxicidad directa que muchos de estos elementos producen en los seres vivos (Kabata-Pendias 1995).

En el caso de las especies vegetales, cada planta es capaz de crecer y reproducirse en forma óptima en un rango de concentración del metal presente en el suelo (Farago 1994); sin embargo, si las concentraciones aumentan por sobre el umbral de tolerancia de la especie, comienzan a producirse efectos de toxicidad, los que conllevan a alteraciones fisiológicas evidenciables en el crecimiento y reproducción de las plantas, pudiendo a veces conducir a la muerte (Sandmann \& Böger 1980, Baker \& Walker 1989, Fernández \& Henríquez 1991, Ernst et al. 1992). De esta forma, todas las plantas responden a incrementos en la concentración de metales en el suelo, sean éstos nutrientes esenciales o no. Sin embargo, se ha encontrado que la concentración total del metal presente en el suelo no se relaciona directamente con los efectos de toxicidad esperados para las especies vegetales sensibles, sino más bien una fracción del total estaría disponible para los seres vivos, la que se ha denominado como concentración biodisponible (e.g., Sauvé et al. 1996, Temminghof et al. 1997, Kabata-Pendias \& Pendias 2000, Badilla et al. 2001, Ginocchio et al. 2002).

Esta fracción biodisponible correspondería a las formas metálicas solubles presentes en la solución suelo (iones y complejos orgánicos de bajo peso molecular) y a formas lábiles adsorbidas a sitios de sorción específicos presentes en la materia orgánica y en las partículas de arcilla constituyentes del suelo, entre otros (Shorrocks \& Alloway 1986, Bourg 1995, Kabata-Pendias 1995, Sauvé et al. 2000), por lo que representaría una fracción pequeña del total. Por ejemplo, se ha determinado que en suelos altamente contaminados con cobre de la zona central de Chile (sobre $1.000 \mathrm{mg} \mathrm{kg}^{-1}$ peso seco), sólo un 0,002\% del cobre total se encuentra como forma iónica en la solución suelo y un $0,04 \%$ se encuentra como formas lábiles, potencialmente utilizables por las plantas (Badilla et al. 2001, Ginocchio et al. 2002). Sin embargo, la fracción biodisponible de contaminantes metálicos no sería constante en suelos con distintas características físico-químicas, ya que muchas de estas variables determinan el grado de solubilización de los metales o la capacidad tamponante del suelo, tales como el $\mathrm{pH}$, el contenido de materia orgánica y la cantidad y tipo de arcillas (Davies 1992, Sauvé et al. 2000). Específicamente, la solubilidad de la mayoría de los metales aumenta al disminuir el $\mathrm{pH}$ del suelo y disminuye al aumentar el contenido de materia orgánica (Bourg 1995, Ginocchio et al. 2002).

Es claro entonces que la naturaleza, dirección y magnitud de la respuesta en la relación plantametal es multifactorial y dependería no sólo de la sensibilidad intrínseca de las especies vegetales, sino que también de diversos factores externos, tales como la intensidad (concentración y duración) de la exposición, del metal involucrado, de la forma química del metal y de las características fisico-químicas del suelo impactado. El objetivo del presente estudio fue evaluar la importancia del tipo de sustrato, en cuanto a su capacidad tamponante de metales, y de la forma química del metal incorporado en la ocurrencia e intensidad de efectos de fitotoxicidad en plántulas de Noticastrum sericeum, una planta herbácea perenne nativa de Chile. El metal seleccionado correspondió al cobre, debido a la importancia de este elemento como contaminante en los suelos de la zona norte y central de Chile (e.g., González \& Ite 1992, González 1994, Ginocchio 2000, Badilla et al. 2001, Ginocchio et al. 2002). Específicamente, la hipótesis puesta a prueba fue que la fitotoxicidad del cobre no depende de la concentración total adicionada al sustrato, sino que debería ser menor al ser incorporado a un sustrato sólido con alta capacidad tamponante (alto contenido de materia orgánica y pH neutro), como el compost comercial, y en una forma química de baja solubilidad, como el óxido de cobre $(\mathrm{CuO})$, que al ser incorporado a un sustrato líquido con baja capacidad tamponante (bajo contenido de materia orgánica y $\mathrm{pH}$ moderadamente ácido) y en una forma química de alta solubilidad, como el sulfato de cobre $\left(\mathrm{CuSO}_{4}\right)$.

\section{MATERIALES Y MÉTODOS}

\section{Material vegetal}

La especie de estudio, Noticastrum sericeum (Less.) Less.ex Phil. (Compositae), corresponde 
a una planta herbácea perenne nativa de Chile central. Fue elegida debido a que mostró ser sensible a incrementos en la concentración de cobre biodisponible en sustratos experimentales (Ginocchio 1999), normalmente utilizados en ensayos estándar de fitotoxicidad; además, las semillas de esta especie representan un buen material para ensayos de laboratorio, ya que presentan un alto porcentaje de germinación (90\%) y una alta tasa de germinación (2 días), lo que permite obtener plántulas en forma rápida y de morfología y tamaño similares.

Semillas de $N$. sericeum, recolectadas en las cercanías de Quintero, Quinta Región de Chile, fueron germinadas en cápsulas de Petri, utilizando papel filtro Whatman $\mathrm{N}^{\circ} 1$ como substrato de inicio y agua desionizada como medio de cultivo. Las cápsulas se sellaron con paraplast y se mantuvieron en una cámara de crecimiento a $22 \pm 2{ }^{\circ} \mathrm{C}$ y fotoperíodo de 12:12 (luz:oscuridad). Las plántulas obtenidas al cabo de cuatro días fueron utilizadas en los ensayos de toxicidad descritos más abajo.

\section{Sustratos de crecimiento}

Se utilizó dos tipos de sustratos, con capacidad contrastante para tamponar la incorporación de metales: (a) un sustrato sólido altamente tamponante y (b) un sustrato líquido con baja capacidad tamponante. El sustrato sólido correspondió a compost comercial (Terra Green) con alto contenido de materia orgánica, $\mathrm{pH}$ neutro, alta capacidad de intercambio catiónico (CIC), alta capacidad de retención de agua y bajas concentraciones de cobre total, cobre soluble $\left(\mathrm{CaCl}_{2}\right)$ y cobre iónico (Tabla 1), previamente tamizado con un tamiz de diámetro de celda de $5 \mathrm{~mm}$. El sustrato líquido correspondió a una solución moderadamente ácida $(\mathrm{pH} \mathrm{5,2)}$ de nitrato de calcio $\left(\mathrm{Ca}\left(\mathrm{NO}_{3}\right)_{2}\right)$ de alta pureza, 2 mmoles $\mathrm{L}^{-1}$, frecuen- temente utilizada en ensayos de fitotoxicidad de metales ya que simula una solución suelo lo más simple posible, que permite mantener estabilizadas las membranas celulares radicales. La solución se preparó con agua desionizada, pH 5,0 y conductividad eléctrica de $8,4 \mathrm{mS} \mathrm{cm}^{-1}$, obtenida de un purificador de agua Option 3, USF Elga, U.K., y nitrato de calcio calidad pro-análisis (Riedel-de Haën).

\section{Formas químicas de cobre}

Se seleccionó para este estudio dos compuestos de cobre con distinta solubilidad: a) sulfato de cobre (II) anhidro pro-análisis (Riedel-de Haën), con un producto de solubilidad $\log \mathrm{K}=2,4 \mathrm{y}$ solubilidad en agua de $143 \mathrm{~g} \mathrm{~L}^{-1}$ a $25^{\circ} \mathrm{C}$ y (b) óxido de cobre (II) anhidro pro-análisis (Riedelde Haën), con un producto de solubilidad $\log \mathrm{K}=$ $-20,5$ y solubilidad muy baja en agua.

\section{Ensayos de toxicidad}

Para cuantificar la importancia de la matriz del sustrato y de la forma química en la toxicidad del cobre sobre plántulas de $N$. sericuem se utilizó un diseño factorial totalmente aleatorizado, con 3 factores, (1) tipo de sustrato de crecimiento, con dos niveles (líquido y sólido), (2) tipo de sal de cobre, con dos niveles $\left(\mathrm{CuO}\right.$ y $\left.\mathrm{CuSO}_{4}\right)$, y (3) concentración de cobre, con tres niveles $(0 ; 0,16$; y 1,6 mmoles $\mathrm{kg}^{-1}$ o mmoles $\left.\mathrm{L}^{-1}\right)$. Cada unidad experimental se replicó 10 veces.

Los sustratos conteniendo distintas concentraciones de óxido o de sulfato de cobre se colocaron en envases de polietileno de $250 \mathrm{~mL}$, previamente lavados con una solución de ácido nítrico al $5 \%$ por $24 \mathrm{~h}$ y enjuagados dos veces con agua desionizada. Se transplantó una plántula de $N$. sericeum de 4 días de edad a cada maceta, teniendo

TABLA 1

Características químicas generales del "compost" comercial (Terra Green) utilizado en los ensayos de toxicidad, previo a la aplicación de cobre; (MOT) materia orgánica total; (CIC) capacidad de intercambio catiónico

General chemical characteristics of the commercial compost (Terra Green) used for the toxicity assays, before copper salt additions; (MOT) total organic matter; (CIC) cation exchange capacity

\begin{tabular}{ccccccccc}
\hline $\begin{array}{c}\mathrm{pH} \\
(\mathrm{agua})\end{array}$ & $\begin{array}{c}\text { MOT } \\
(\%)\end{array}$ & $\begin{array}{c}\mathrm{CIC} \\
\left(\mathrm{meq} 100 \mathrm{~g}^{-1}\right)\end{array}$ & $\begin{array}{c}\mathrm{Zn}_{\text {total }} \\
\left(\mathrm{mg} \mathrm{kg}^{-1}\right)\end{array}$ & $\begin{array}{c}\mathrm{Cd}_{\text {total }} \\
\left(\mathrm{mg} \mathrm{kg}^{-1}\right)\end{array}$ & $\begin{array}{c}\mathrm{Pb}_{\text {total }} \\
\left(\mathrm{mg} \mathrm{kg}^{-1}\right)\end{array}$ & $\begin{array}{c}\mathrm{Cu}_{\text {total }} \\
\left(\mathrm{mg} \mathrm{kg}^{-1}\right)\end{array}$ & $\begin{array}{c}\mathrm{Cu}_{\mathrm{CaCl} 2} \\
\left(\mathrm{mg} \mathrm{kg}^{-1}\right)\end{array}$ & $\begin{array}{c}\mathrm{Cu}_{\text {ionico }} \\
\left(\mathrm{mg} \mathrm{kg}^{-1}\right)\end{array}$ \\
\hline 7,87 & 53,8 & 52,3 & 57,92 & 0,03 & 5,28 & 38,04 & 0,018 & $2,5 \times 10^{-10}$ \\
\hline
\end{tabular}


la precaución de elegir plántulas con morfologías radicales y del vástago lo más similares posibles. En el caso del sustrato líquido, las plántulas se sostuvieron en la superficie utilizando láminas de poliestireno perforadas, a las cuales se ancló el cuello de la plántula con un anillo de poliestireno. Las macetas que contenían substrato sólido fueron perforadas en la base para permitir el escurrimiento del exceso de agua de riego.

Las macetas experimentales se mantuvieron en el invernadero del Departamento de Ecología de la P. Universidad Católica de Chile, bajo condiciones controladas de temperatura $\left(22 \pm 3{ }^{\circ} \mathrm{C}\right)$, humedad relativa del aire $(50 \%)$, radiación de 3.000 Lux y fotoperíodo (12:12, luz:oscuridad). Las macetas con sustrato sólido se regaron con $33 \mathrm{~mL}$ de agua desionizada cada 2 días, para mantenerlas a un 80 $\%$ de capacidad de campo, mientras que la solución líquida se reemplazó cada 2 días, con el fin de mantener las características químicas generales y las concentraciones de cobre experimentales constantes. Transcurrido 22 días se cosechó las plántulas contenidas en las macetas experimentales, extrayendo tanto el vástago como el sistema radical. Las variables respuesta analizadas fueron el número de hojas producidas (sin considerar los cotiledones), la longitud de la raíz principal, el número de raíces secundarias y el peso seco de la biomasa radical y aérea. Para las determinaciones de biomasa, las plántulas se lavaron con agua desionizada, se separaron en vástago y raíz, se secaron en estufa de aire forzado a $60{ }^{\circ} \mathrm{C}$ por $48 \mathrm{~h}$ y se pesaron.

\section{Análisis estadístico}

Para determinar diferencias estadísticamente significativas entre los distintos tratamientos se realizó análisis de varianza (ANDEVA) de tres vías, para cada una de las variables respuesta determinadas $(\alpha=0,01)$. Se empleó la prueba HSD ("honest signifficant difference") de Tukey como prueba a posteriori (Steel \& Torrie 1985). Para realizar estos análisis se empleó el paquete estadístico Statistica para Windows (StatSoft 1993).

\section{RESULTADOS}

\section{Respuestas a nivel del vástago}

La producción de hojas nuevas se redujo en forma significativa sólo en las plántulas crecidas en sustrato líquido enriquecido con sulfato de cobre $\left(0,16\right.$ y 1,6 mmoles $\left.\mathrm{kg}^{-1}\right)$ y con concentraciones

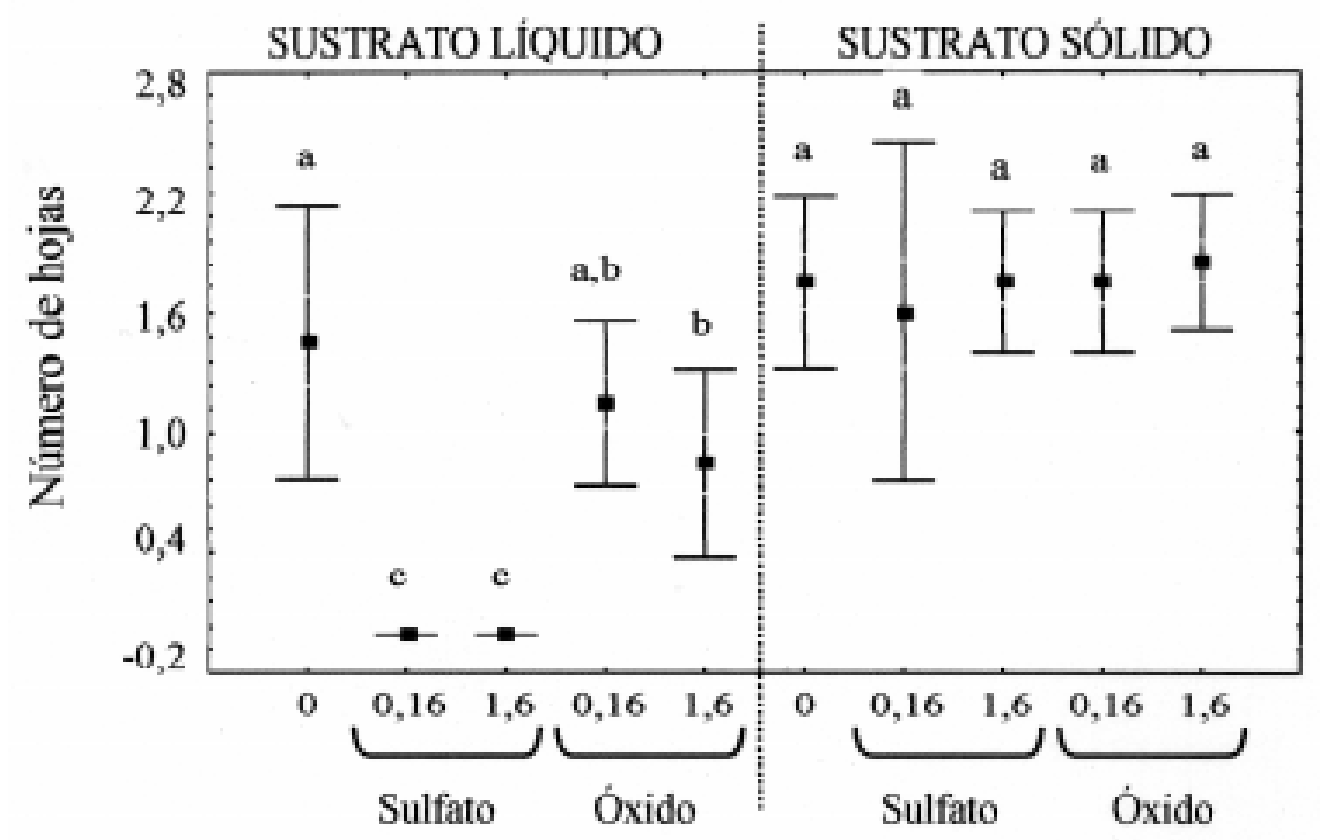

Fig. 1: Número de hojas producidas por plántulas de Noticastrum sericeum sometidas a concentraciones crecientes (control; $0 ; 16$ y 1,6 mmoles $\mathrm{L}^{-1}$ o mmoles $\mathrm{kg}^{-1}$ ) de sulfato y óxido de cobre, en sustrato líquido y sólido, por 22 días. Letras diferentes indican diferencias significativas entre los tratamientos. Se indica el promedio (ם) y desviación estándar (I).

Number of leaves produced by Noticastrum sericeum seedlings exposed to increasing concentrations (control, 0.16 and 1.6 mmol L-1 or mmol kg${ }^{-1}$ ) of copper sulphate and oxide, in solid and liquid growing media, after 22 days. Different letters indicate significant differences between treatments. The mean

$\square$ ) and standard deviation (I) are shown. 
elevadas de óxido de cobre (1,6 mmoles $\left.\mathrm{kg}^{-1}\right)$, pero no se afectó en ninguno de los sustratos sólidos enriquecidos con sales de cobre, a ninguna de las concentraciones utilizadas (Fig. 1). En las plántulas afectadas, la producción de hojas nuevas fue nula, conservando sólo los cotiledones. Una reducción significativa en la biomasa media del vástago se observó en las plántulas crecidas en sustrato líquido adicionado con 1,6 mmoles $\mathrm{L}$ ${ }^{1}$ de sulfato de cobre, mostrando este tratamiento diferencias significativas con las plántulas de los tratamientos control y 0,16 mmoles $\mathrm{kg}^{-1}$ de óxido de cobre del sustrato sólido (Fig. 2). Los vástagos de las plántulas crecidas en sustrato líquido adicionado con 0,16 y 1,6 mmoles $\mathrm{L}^{-1}$ de sulfato de cobre fueron los únicos que mostraron síntomas visibles de estrés por toxicidad transcurrido 22 días de experimentación, al presentar cotiledones marchitos, cloróticos y enrojecidos. Además, se observó necrosis en el meristema apical de las plántulas crecidas en solución líquida enriquecida con 1,6 mmoles $\mathrm{L}^{-1}$ de sulfato de cobre.

\section{Respuestas a nivel de la raíz}

Las cargas metálicas adicionadas al sustrato líquido, ya sea en forma de sulfato o de óxido de cobre, resultaron en plántulas con raíces primarias significativamente más cortas que los tratamientos equivalentes en sustrato sólido (Fig. 3), indicando efectos negativos en el crecimiento radical. Las raíces primarias de las plántulas crecidas en los tratamientos control eran largas y delgadas, con una coloración blanquecina, mientras que las raíces primarias de las plántulas mantenidas en soluciones líquidas enriquecidas con cobre mostraron atrofia y necrosis del meristema terminal, síntomas característicos de intoxicación por concentraciones excesivas de cobre. En el caso de las plántulas crecidas en el sustrato sólido, no se detectó efectos negativos significativos sobre la longitud radical final, en ninguno de los tratamientos experimentales ensayados (Fig. 3), indicando un efecto tampón adecuado de este tipo de sustrato a las cargas metálicas adicionadas.

El mismo patrón de respuesta cuantificado en la longitud de la raíz principal se detectó en el número de raíces secundarias producidas (Fig. 4) y en la biomasa radical (Fig. 5). La producción de raíces secundarias en las plántulas crecidas en los sustratos líquidos enriquecidos con sulfato y óxido de cobre fue completamente inhibida, independientemente de la concentración de cobre adicionada, en comparación con los otros tratamientos (Fig. 4). Este resultado evidencia síntomas de

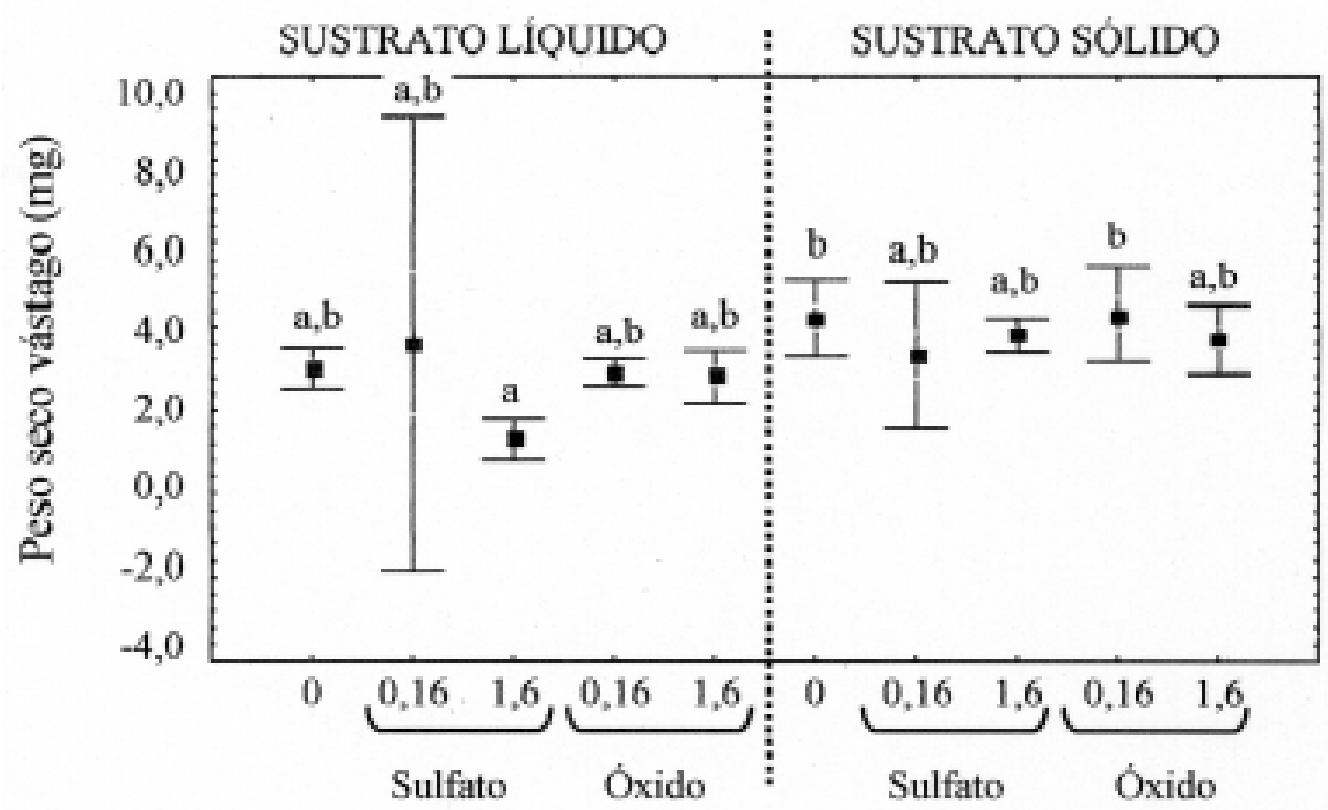

Fig. 2: Biomasa del vástago (peso seco) de plántulas de N. sericeum sometidas a concentraciones crecientes (control, 0, 16 y 1,6 mmoles $\mathrm{L}^{-1} \mathrm{o}_{\text {mmoles }} \mathrm{kg}^{-1}$ ) de sulfato y óxido de cobre, en sustrato líquido y sólido, por 22 días. Letras diferentes indican diferencias significativas entre los tratamientos. Se indica el promedio ( $\mathbf{\square})$ y desviación estándar (I).

Shoot biomass (dry weight) of $N$. sericeum seedlings exposed to increasing concentrations (control, 0.16 and $1.6 \mathrm{mmol} \mathrm{L}^{-1}$ or $\mathrm{mmol} \mathrm{kg} \mathrm{kg}^{-1}$ ) of copper sulphate and copper oxide, in solid and liquid growing media, after 22 days. Different letters indicate significant differences between treatments. The mean (ם) and standard deviation (I) are shown. 


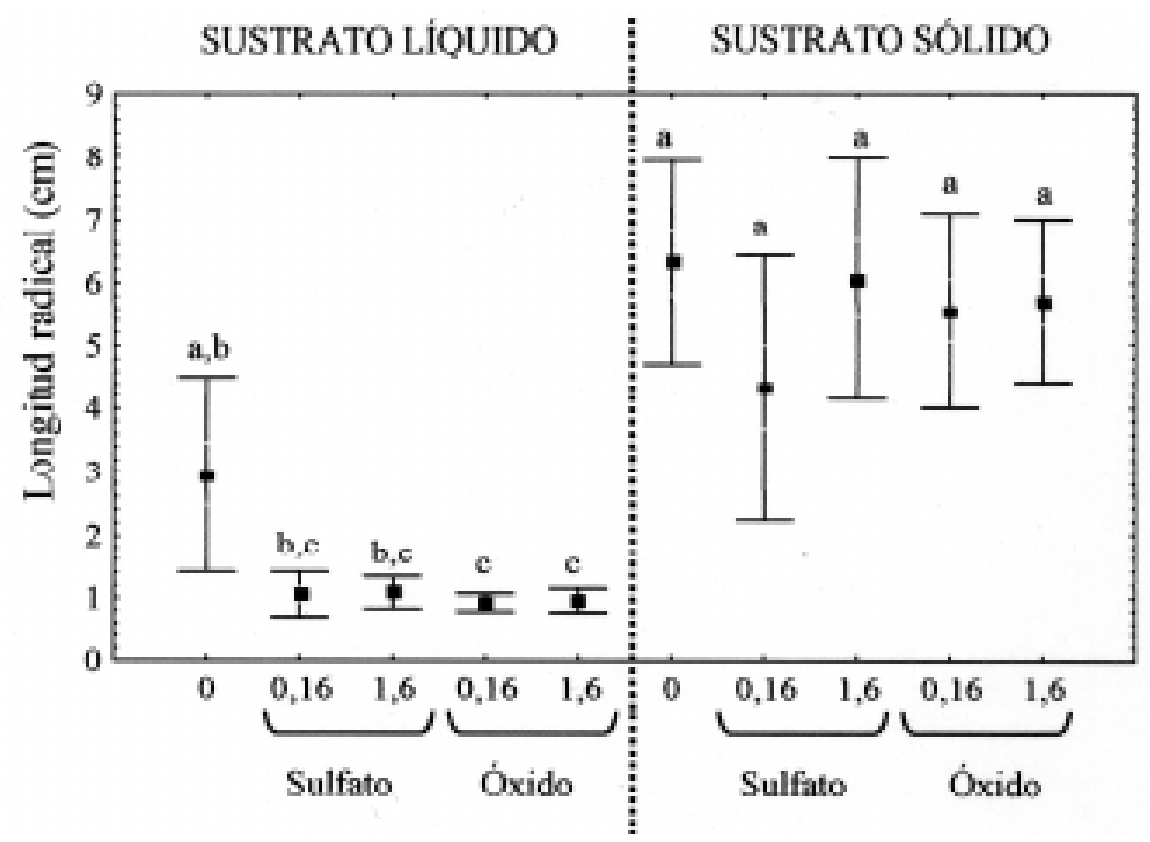

Fig. 3: Longitud de la raíz principal de plántulas de $N$. sericeum sometidas a concentraciones crecientes (control; 0,16 y 1,6 mmoles $\mathrm{L}^{-1}$ o mmoles $\mathrm{kg}^{-1}$ ) de sulfato y óxido de cobre, en sustrato líquido y sólido, por 22 días. Letras diferentes indican diferencias significativas entre los tratamientos. Se indica el promedio ( $\square$ ) y desviación estándar (I).

Length of primary root of $N$. sericeum seedlings exposed to increasing concentrations (control, 0.16 and $1.6 \mathrm{mmol} \mathrm{L}^{-1}$ or

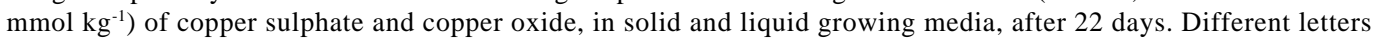
indicate significant differences between treatments. The mean ( $\square$ ) and standard deviation (I) are shown.

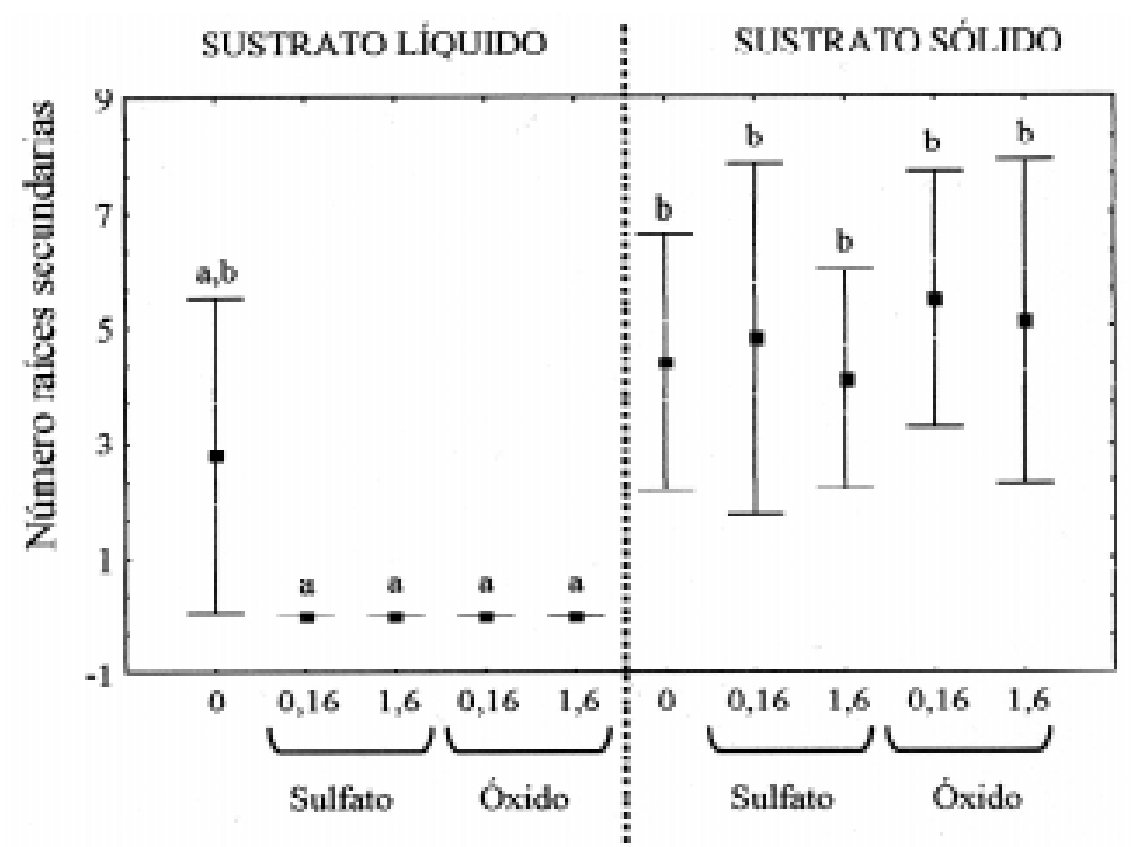

Fig. 4: Número de raíces secundarias producidas por plántulas de N. sericeum sometidas a concentraciones crecientes (control; 0,16 y 1,6 mmoles $\mathrm{L}^{-1}$ o mmoles kg-1) de sulfato y óxido de cobre, en sustrato líquido y sólido, por 22 días. Letras diferentes indican diferencias significativas entre los tratamientos. Se indica el promedio (ם) y desviación estándar (I).

Number of secondary roots produced by $N$. sericeum seedlings exposed to increasing concentrations (control, 0.16 and 1.6 mmol L-1 or mmol kg${ }^{-1}$ ) of copper sulphate and copper oxide, in solid and liquid growing media, after 22 days. Different letters indicate significant differences between treatments. The mean $(\boldsymbol{\square})$ and standard deviation (I) are shown. 


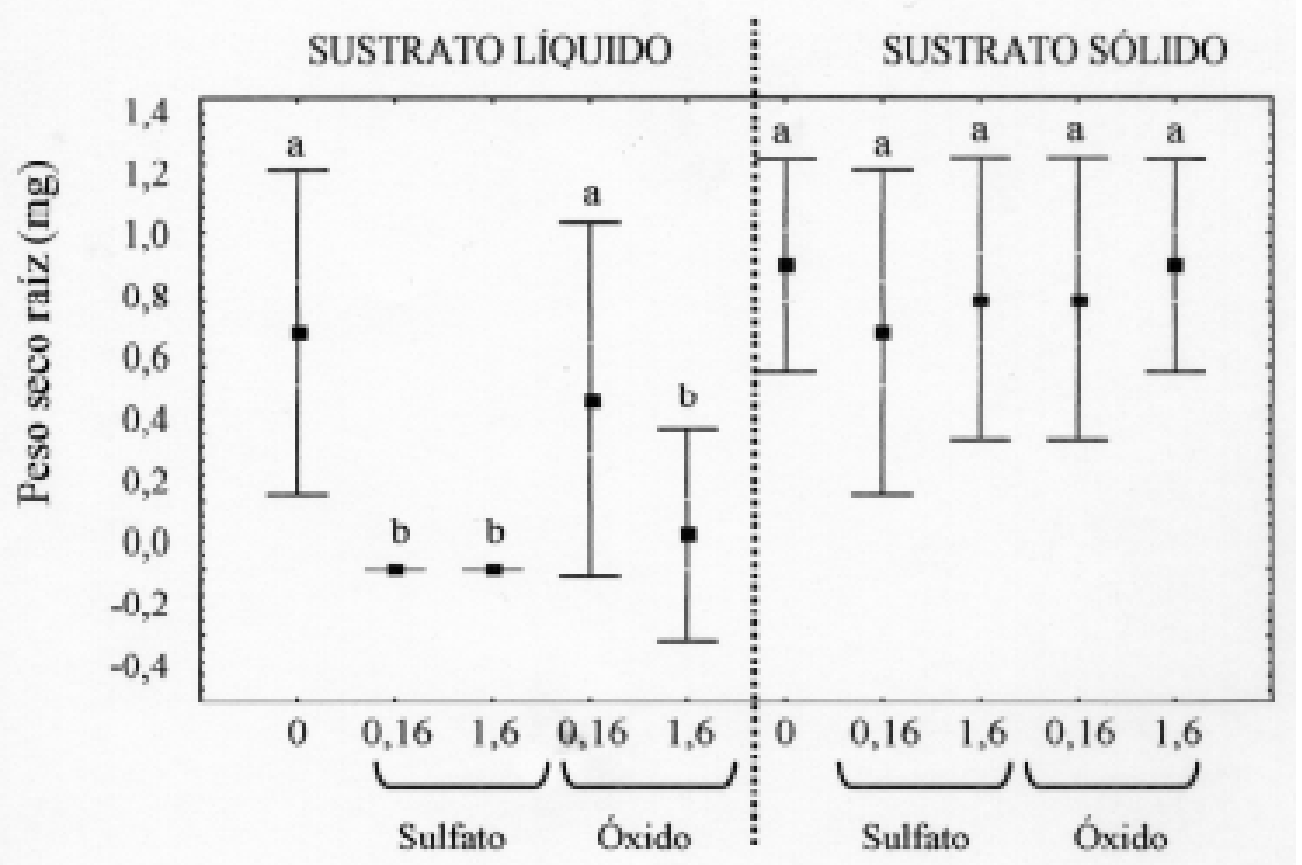

Fig. 5: Biomasa radical (peso seco) de plántulas de $N$. sericeum sometidas a concentraciones crecientes (control; 0,16 y 1,6 mmoles $\mathrm{L}^{-1} \mathrm{o}$ mmoles $\mathrm{kg}^{-1}$ ) de sulfato y óxido de cobre, en sustrato líquido y sólido, por 22 días. Letras diferentes indican diferencias significativas entre los tratamientos. Se indica el promedio (ם) y desviación estándar (I).

Root biomass (dry weight) of $N$. sericeum seedlings exposed to increasing concentrations (control, 0.16 and $1.6 \mathrm{mmol} \mathrm{L}^{-1}$ or $\mathrm{mmol} \mathrm{kg} \mathrm{k}^{-1}$ ) of copper sulphate and copper oxide, in solid and liquid growing media, after 22 days. Different letters indicate significant differences between treatments. The mean (ם) and standard deviation (I) are shown.

toxicidad por cantidades excesivas de cobre, al inhibirse la actividad celular de los meristemas axilares. No se detectó inhibición en la producción de raíces secundarias en ninguno de los tratamientos en los que se utilizó sustrato sólido como medio de crecimiento (Fig. 4).

La biomasa radical producida por las plántulas en experimentación varió significativamente con el tipo de sustrato de crecimiento, sal de cobre y concentración de cobre agregadas (Fig. 5). La biomasa radical media producida en el sustrato sólido fue significativamente mayor que la biomasa media producida en el sustrato líquido. La adición de sulfato de cobre o de óxido de cobre no inhibió la producción de biomasa radical en las plántulas crecidas en el sustrato sólido, a ninguna de las concentraciones adicionadas (Fig. 5). Sin embargo, la adición de sulfato de cobre $\left(0,16\right.$ y 1,6 mmoles $\left.\mathrm{L}^{-1}\right)$ o de óxido de cobre (1,6 mmoles $\left.\mathrm{L}^{-1}\right)$ al sustrato de crecimiento líquido, inhibió significativamente la producción de biomasa radical (Fig. 5).

\section{DISCUSIÓN}

Mediante este estudio se logró determinar los efectos fitotóxicos producidos sobre $N$. sericeum por la adición de formas químicas de cobre con distinta solubilidad en agua, a sustratos con capacidades tamponantes contrastantes, principalmente en términos del contenido de materia orgánica y $\mathrm{pH}$. Los resultados nos permiten apoyar la hipótesis que la respuesta en la relación planta-cobre no está determinada por la concentración total de metal adicionada al sustrato, sino que corresponde más bien a una respuesta multifactorial que depende de la forma química en la cual se encuentre este elemento y de la capacidad tamponante del sustrato. Se ha demostrado que el desplazamiento ó movilidad de los metales en el suelo depende de las concentraciones y afinidades relativas de los iones involucrados por las superficies sólidas del suelo, la formación de complejos a diferentes pH y de la fuerza iónica (Barceló \& Poschenrieder 1989). Adicionalmente, la amortiguación que ofrezca un suelo a la contaminación con metales pesados dependerá del contenido de materia orgánica, $\mathrm{pH}$, CIC y de las propiedades químicas de los metales. De acuerdo a Gambrell et al. (1986), los factores edáficos que más regulan la solubilidad y movilidad de los metales en suelos son el pH, las condiciones redox, la presencia de substancias orgánicas con capacidad complejante y el tipo y cantidad de minerales de 
arcilla, así como también los factores climáticos que potencian el estrés metálico.

Tal como se esperaba, los efectos de fitotoxicidad producidos por el sulfato de cobre fueron más marcados que los producidos por el óxido de cobre, fundamentalmente en el sustrato poco tamponante (solución de nitrato de calcio), debido a la mayor solubilidad de este compuesto en agua, en un medio de menor $\mathrm{pH}$ y en ausencia de materia orgánica. Al encontrarse una mayor concentración de iones cobre en el sustrato líquido, mayor podría ser la absorción de este elemento por las raíces de las plántulas, lo que podría dar cuenta de la toxicidad observada en las plántulas de estudio, principalmente a nivel radical.

Se ha demostrado que los efectos primarios de toxicidad por metales pesados se producen en las raíces, las que muestran una menor elongación después de ser expuestas a estos elementos (Marschner 1986, Larcher 1995, Lambers et al. 1998), por lo que es esperable que el crecimiento radical haya sido más afectado que el crecimiento del vástago en $N$. sericeum, para una misma carga metálica y tipo de sal de cobre agregada. Los síntomas de toxicidad descritos en la literatura para esta estructura vegetal tienen que ver con un menor crecimiento de la raíz principal por muerte del meristema apical de la raíz principal, estimulación de la formación de raíces secundarias y posterior inhibición del meristema apical de las raíces secundarias cuando la concentración de metal supera el límite de tolerancia (Marschner 1986, Baker 1987, Kabata-Pendias 1995, Cook et al. 1997). Sin embargo, el aumento del peso seco radical es menos afectado que la longitud radical, lo que origina raíces de aspecto achaparrado (Brune et al. 1994), tal como se observó en las raíces de las plántulas más afectadas de $N$. sericeum.

\footnotetext{
${ }^{1}$ ALLEN HE \& Y YIN (1997) Natural remediation of metals in soils: adsorption: 5. Proceedings of the Society of Environmental Toxicology and Chemistry, SETAC, Eighteenth Annual Meeting, San Francisco, California, U.S.A.

${ }^{2}$ ALLEN HE, B SHI, MT GRASSI, H MA, Y ZONG \& Y YIN (1997) Chemistry of copper bioavailability. II: adsorption to suspended particulate matter and complexation by DOM: 9495. Proceedings of the Society of Environmental Toxicology and Chemistry, SETAC, Eighteenth Annual Meeting, San Francisco, California, U.S.A.
}

Una vez sobrepasados los mecanismos de tolerancia al exceso de cobre acumulado en las raíces, la fisiología general de la planta sería afectada, produciéndose translocación excesiva de este elemento al vástago, donde afectaría negativamente la fotosíntesis y otras funciones celulares que resultan en síntomas visibles de toxicidad, tales como menor desarrollo del vástago, clorosis y enrojecimiento de las hojas (Baker 1987, Fernández \& Henríquez 1991, Ernst et al. 1992). En este estudio, síntomas de toxicidad a nivel del vástago sólo se cuantificaron en el número de hojas nuevas producidas al exponer las plántulas de $N$. sericeum a sulfato de cobre y a altas concentraciones de óxido de cobre, en solución líquida. Estas plántulas también mostraron síntomas visibles de intoxicación por cobre, con vástagos rojizos y cloróticos.

La gran capacidad tamponante del sustrato sólido utilizado, en términos de su contenido de materia orgánica y $\mathrm{pH}$, habría determinado concentraciones muy baja de cobre disponible para la absorción radical, reflejándose ésto en la ausencia de fitoxicidad observada en $N$. sericuem, tanto en sus estructuras subterráneas como aéreas. El alto porcentaje de materia orgánica presente en el "compost" comercial utilizado $(53,8 \%)$ y su $\mathrm{pH}$ neutro $(7,9)$ constituyen parámetros básicos en determinar esta alta capacidad tamponante. Debido a la alta capacidad de la materia orgánica para formar complejos estables con los iones metálicos, la relación entre la materia orgánica del suelo y los metales es muy importante, especialmente en ambientes alcalinos, donde la afinidad es aún mayor (Yin et al. 1996). De acuerdo a Alloway (1995a), una de las asociaciones materia orgánica-metal más estrecha se da con el $\mathrm{Cu}$, lo que redunda en una fuerte disminución de su potencial tóxico (Allen \& Yin $1997^{1}$, Allen et al. 1997²). Por otra parte, el pH es otro factor importante en gobernar la solubilidad de los metales en el suelo y, por ende, su movilidad y disponibilidad para las plantas. Específicamente, a medida que disminuye el pH aumenta la solubilidad del cobre presente en el suelo (Alloway 1995a). De esta forma, suelos con $\mathrm{pH}$ ácidos promueven un aumento de la toxicidad de metales, mientras que suelos con $\mathrm{pH}$ neutro muestran menor toxicidad debido a la menor solubilidad de los metales presentes (Gambrell et al. 1991, Kabata-Pendias 1993, Ashtab \& Yel'nikov 1996, Kitao et al. 1997).

Finalmente, es importante destacar que para todas las variables respuestas medidas no se encontró diferencias significativas entre los tratamientos control del sustrato líquido y sólido, por lo que los efectos de toxicidad por cobre eviden- 
ciados en las plántulas crecidas en el sustrato líquido se atribuyen principalmente a los efectos del tipo de sal adicionada y la concentración de cobre probadas y no a diferencias nutricionales de los sustratos de crecimiento. Los ensayos experimentales utilizaron plántulas juveniles de $N$. sericeum y no plántulas de edad más avanzada o plantas adultas, debido a que su crecimiento sería sustentado en mayor parte por las reservas nutritivas almacenadas en los cotiledones, más bien que sólo por los aportes de recursos minerales externos.

\section{AGRADECIMIENTOS}

Este estudio fue financiado por los proyectos FONDECYT 1000750 a R. Ginocchio y CIMMICA suelos a R. Ginocchio y Patricio Rodríguez. Los autores agradecen la colaboración de Elena Bustamante y Yasna Silva, del Centro de Investigaciones Mineras y Metalúrgicas, CIMM, en los análisis químicos cuantitativos realizados en este estudio.

\section{LITERATURA CITADA}

ALFARO LA (1988) Cambio en el uso del suelo agrícola por efecto de la contaminación ambiental. Caso de estudio: comuna de Puchuncaví. Tesis de Grado Instituto de Geografía, Pontificia Universidad Católica de Chile, Santiago, Chile. 135 pp.

ALLEN H, CH HUANG, G BAILEY \& A BOWERS (1995) Metal speciation and contamination of soil. Lewis Publishers, CRC Press, Boca Raton, Florida. 384 pp.

ALLOWAY BJ (1995a) Soil process and the behavior of the heavy metals. En: Alloway BJ (ed) Heavy metals in soils: 11-37. Blackie Academic and Professional, Londres, United Kingdom. 339 pp.

ASHTAB IV \& II YEL'NICOV (1996) Evaluation of Zn levels in Chernozems from the elemental composition of plants. Eurasian Soil Science 28: 230-239.

BADILLA-OHLBAUM R, R GINOCCHIO, PH RODRÍGUEZ, A CÉSPEDES, S GONZÁLEZ, HE ALLEN \& GE LAGOS (2001) Effect of soil copper content on copper load of selected crop plants in central Chile. Environmental Toxicology and Chemistry 20: 2749-2757.

BAKER AJM (1987) Metal tolerance. New Phytologist 106: 93-11.

BAKER AJM \& PL WALKER (1989) Physiological responses of plants to heavy metals and the quantification of tolerance and toxicity. Chemical Specialization and Bioavailability 1: 7-17.

BARCELO J \& CH POSCHENRIEDER (1989) Estrés vegetal inducido por metales pesados. Investigación y Ciencia 145: 55-63.
BOURG ACM (1995) Speciation of heavy metals in soils and groundwater. En: Salomons W, U Förstner \& P Mader (eds) Heavy metals. Problems and Solutions: 3-17. Springer-Verlag, Berlin, Germany. 412 pp.

BRUNE A， URBACH W \& DIETZ KJ (1994) Compartmentation and transport of zinc in barley primary leaves as basic mechanisms involved in zinc tolerance. Plant Cell Environment 17: 153-162.

COOK CM, A KOSTIDOU, E VARDAKA \& T LANARAS (1997) Effects of copper on the growth, photosynthesis and nutrient concentrations of Phaseolus plants. Photosynthetica 34: 179-193.

DAVIES BE (1992) Interrelationships between soil properties and the uptake of cadmium, copper, lead, and zinc from contaminated soils by radish (Raphanus sativus L.). Water, Air and Soil Pollution 63: 331342 .

DUDKA S, M PIOTROWSKA, A CHLOPECKA \& $\mathrm{T}$ WITEK (1995) Trace metal contamination of soils and crop plants by mining and smelting industry in Upper Silesia, south-west Polland. Journal of Geochemical Exploration 52: 237-250.

ERNST WH, J VERKLEIJ \& W SCHAT (1992) Metal tolerance in plants. Acta Botanica Netherland 41: 229-248.

FARAGO M (1994) Plants and the chemical elements: biochemistry, uptake, tolerance and toxicity. $\mathrm{VCH}$ Verlasgesellschaft, Weinheim, Germany. 292 pp.

FERNÁNDEZ JC \& FS HENRÍQUEZ (1991) Biochemical, physiological, and structural effects of excess copper in plants. Botanical Review 57: 246-273.

GAMBRELL RP, S LANDES, CN REDDY \& WH PATRICK (1986) Soil phytosicochemical parameters affecting metal availability in sludge-amended soils. EPA Project Summary, Research and Development, EPA/600/S2-85/123, U.S.A.

GAMBRELL RP, JB WIESEPAPE, WH PATRICK \& MC DUFF (1991) Effects of $\mathrm{pH}$, redox, and salinity on metal release from a contaminated sediments. Water, Air and Soil Pollution 57-58: 359-367.

GINOCCHIO R (1999) Copper tolerance testing on plant species growing near a copper smelter in central Chile. En: Wenzel WW, DC Adriano, B Alloway, HE Doner, C Keller, NW Lepp, M Mench, R Naidu \& GM Pierzynski (eds) Proceedings of the Fifth International Conference on the Biogeochemistry of Trace Elements: 1156-1157. International Society for Trace Element Research, Viena, Austria.

GINOCCHIO R (2000) Effects of a copper smelter on a grassland community in the Puchuncaví valley, Chile. Chemosphere 41: 15-23.

GINOCCHIO R, PH RODRÍGUEZ, R BADILLAOHLBAUM, HE ALLEN \& G LAGOS (2002) Effect of soil copper content and $\mathrm{pH}$ on copper uptake of selected vegetables grown under controlled conditions. Environmental Toxicology \& Chemistry 21: 117-125.

GONZÁLEZ S (1994) Estado de la contaminación de los suelos en Chile. En: Espinoza G, P Pisani, L Contreras \& P Camus (eds) Perfil ambiental de Chile: 199-234. Comisión Nacional del Medio Ambiente, Santiago, Chile. 569 pp. 
GONZÁLEZ S \& R ITE (1992) Acumulación metálica en suelos del área bajo influencia de las chimeneas industriales de Ventanas (Provincia de Valparaíso, V Región). Agricultura Técnica (Chile) 50: 214-219.

KABATA-PENDIAS A (1993) Behavioural properties of trace metals in soils. Applied Geochemistry (supplement issue) 2: 3-9.

KABATA-PENDIAS A (1995) Agricultural problems related to extensive trace metal contents of soils. En: Salomons W, U Förstner \& P Mader (eds) Heavy metals, problems and solutions: 3-18. SpringerVerlag, Berlin, Germany. $412 \mathrm{pp}$.

KABATA-PENDIAS A \& H PENDIAS (2000) Trace elements in soils and plants. CRC Press, An Arbor, Michigan. 432 pp.

KIM K-H \& S-H KIM (1999) Heavy metal pollution of agricultural soils in central regions of Korea. Water, Air and Soil Pollution 111: 109-122.

KITA N, TT LE \& T KOIKE (1997) Comparison of photosynthetic responses to manganese toxicity of deciduous broad-leaved trees in northern Japan. Environmental Pollution 97: 113-118.

LAMBERS H, FS CHAPIN III \& TL PONS (1998) Plant physiological ecology. Springer-Verlag, New York, New York. 540 pp.

LARCHER W (1995) Physiological plant ecology. Springer-Verlag, Berlin, Germany. 506 pp.

MARSCHNER H (1986) Mineral nutrition of higher plants. Academic Press, London, United Kingdom. 674 pp.
SANDMANN G \& P BÖGER (1980) Copper-mediated lipid peroxidation processes in photosynthetic membranes. Plant Physiology 66: 797-800.

SAUVÉ S, N COOK, WH HENDERSHOT \& MB MCBRIDE (1996) Linking plant tissue concentrations and soil copper pools in urban contaminated soils. Environmental Pollution 94: 153-157.

SAUVÉ S, W HENDERSHOT \& HE ALLEN (2000) Solidsolution partitioning of metals in contaminated soils: dependence on $\mathrm{pH}$ and total metal burden. Environmental Science and Technology 34: 11251131.

SHORROCKS VM \& BJ ALLOWAY (1986) Copper in plant, animal and human nutrition. Copper Development Association, Report TN 35. Orchard House, London United Kingdom. 84 pp.

STATSOFT (1993) Statistica for Windows, version 4.5. StatSoft Inc., Tulsa, Oklahoma.

STEEL GD \& JH TORRIE (1985) Bioestadística: principios y procedimientos. McGraw-Hill, Bogotá, Colombia. $622 \mathrm{pp}$.

TEMMINGHOF EJM, ACC PLETTE, ZEE SEATMVD \& WHV RIEMSDIJK (1997) Speciation of heavy metals in soil in relation to availability and mobility. Recent Research Development in Soil Science 1: 55-65.

YIN Y, HE ALLEN, Y LI, CP HUANG \& PF SANDERS (1996) Adsorption of mercury (II) by soil: effects of $\mathrm{pH}$, chloride and organic matter. Journal of Environmental Quality 25: 837-844. 